122

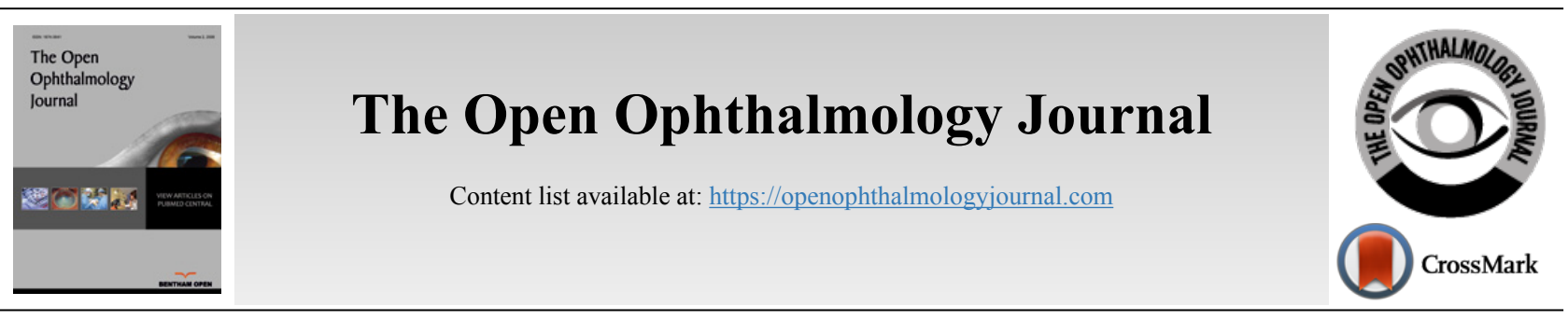

RESEARCH ARTICLE

\title{
Epidemiology of Orbital Fractures in a Large Hospital in the Netherlands: Results of Implementation of a Multidisciplinary Orbital Trauma Team
}

\author{
Tamar J. A. Kooger ${ }^{1, *}$, Maurits V. Joosse ${ }^{2}$, Ellen M. van Minderhout ${ }^{3}$, Kenneth Hergaarden ${ }^{4}$ and Yu-Lan Khouw ${ }^{5}$ \\ ${ }^{1}$ Medical Intern at the Haaglanden Medical Centre Lijnbaan 32, 2512 VA Den Haag, The Netherlands \\ ${ }^{2}$ Department of Ophthalmology, Haaglanden Medical Centre, Lijnbaan 32, 2512 VA Den Haag, The Netherlands \\ ${ }^{3}$ Department of Orthoptist, Haaglanden Medical Centre, Lijnbaan 32, 2512 VA Den Haag, The Netherlands \\ ${ }^{4}$ Department of radiology, Radiology resident Leiden University Medical Centre, Albinusdreef 22333 ZA Leiden, The Netherlands \\ ${ }^{5}$ Department of Oral-Maxillofacial Surgery, Oral and Maxillofacial Surgeon Haaglanden Medical Centre, Lijnbaan 32 , 2512 VA Den Haag, The \\ Netherlands
}

\begin{abstract}
:
Introduction:

This study aims to describe the epidemiology, aetiology and therapy of orbital fractures in a large municipal hospital in The Hague, the Netherlands, and to demonstrate the effectiveness of an integrated approach of orbital trauma care with a team consisting of an ophthalmologist, an orthoptist and an oral and maxillofacial (OMF) surgeon.

Methods:

We obtained data from patients with an orbital fracture between June 2015 until July 2018. Primary outcomes were the classification of orbital fractures and treatment (conservative or surgical).

Results:

182 patients were included in this study. The most common type of orbital fracture was an isolated orbital floor fracture ( $\mathrm{n}=56,30.8 \%)$. In most cases, therapy was conservative $(69.2 \%)$. When surgical repair was indicated, a polydioxanone (PDS) plate was most commonly used (11.5\%). The main cause of an orbital fracture was a fall accident (38.5\%), followed by assault (32.4\%). 32.4\% of the patients were intoxicated with alcohol.

\section{Conclusion:}

In our study population, almost $70 \%(69.2 \%)$ of the patients were treated conservatively in our study. Compared to the international literature, this percentage is considerably higher than in other studies. In our opinion, our integrated approach to orbital trauma has led to a justifiable reduction of the proportion of cases receiving surgical intervention in orbital fractures. We would like to advocate the implementation of a similar team in other hospitals to improve the quality and cost-effectiveness of treatment in patients with orbital fractures.
\end{abstract}

Keywords: Orbital fracture, Orbital trauma, Maxillofacial trauma, Epidemiology, Orbital care, Integrated approach.

\begin{tabular}{|l|l|l|r} 
Article History & Received: February 6, 2021 & Revised: April 11, 2021 & Accepted: May 6, 2021
\end{tabular}

\section{INTRODUCTION}

Yearly, on average, 54.000 patients visit the emergency room (ER) of the Haaglanden Medical Centre (HMC) Westeinde in the Hague (the Netherlands). From June 2015 until July 2018, nearly 4600 patients were triaged in the ER with a diagnosis of 'maxillofacial injury' and 'head injury'.

\footnotetext{
* Address correspondence to this author at Medical Intern at the Haaglanden Medical Centre Lijnbaan 32, 2512 VA Den Haag, The Netherlands; Tel: +31 651791992; Fax: +31 703125993; E-mail: tamarkooger@gmail.com
}

Over the last years, the diagnostic measures to analyse neurocranial trauma have become more optimized in our hospital because of the protocolized usage of computed tomography $(\mathrm{CT})$ at the ER with each maxillofacial trauma. Moreover, the introduction of emergency physicians in the ER has raised a greater awareness of orbital fractures.

Orbital fractures may be isolated (often broadly called 'blow-out' fractures); however, many orbital fractures are part of the more extensive maxillofacial injury. In the United States, about $3 \%$ of the ER visits are related to the trauma of the eye or 
orbit [1]. A large European study showed that approximately $16 \%$ of maxillofacial fractures were orbital blow-out fractures [2]. The majority of orbital wall fractures occur in males in their thirties and are often the result of blunt maxillofacial trauma [3 - 5]. In adults, the main cause of orbital trauma is assault, followed by motor vehicle accidents and falls [3 - 9]. In paediatric patients, activities in daily life and sports injuries are more common $[10,11]$. Typical symptoms of orbital wall fractures are periocular hematoma (and/or oedema), tenderness of the orbital rim, diplopia and reduced sensibility in the area of the infraorbital nerve [3].

Alcohol consumption may cause patients to be predisposed to assault or motor vehicle accidents, which can cause an orbital fracture. Alcohol consumption in young adults in the Netherlands is decreasing; however, it is still above the international average of alcohol consumption [12]. Literature reveals that the risk of ocular trauma is increased in patients with alcohol intoxication [13]. Also, in 20-25\% of patients with maxillofacial fractures, including orbital fractures, alcohol intoxication has been noted $[6,14]$.

In our hospital, the ophthalmologist and oral maxillofacial (OMF) surgeon are the primary specialists to analyse and treat orbital fractures. However, the number and type of specialists involved in the treatment of orbital fractures can differ between countries as well as between hospitals $[15,16]$. The literature is still inconclusive regarding when and/or in which cases surgical repair of orbital fractures is indicated. A recent study shows that the majority of patients with conservatively treated orbital blowout fractures show spontaneous radiological improvement [17]. Early surgical intervention in less than 24 hours (lateral canthotomy or orbital floor repair) in orbital fractures is primarily indicated when vision is threatened because of compression or traction of the optical nerve (because of a retrobulbar hematoma) and because of entrapment of the eye musculature [3,18]. The latter mostly occurs in paediatric patients. This fracture is commonly known as a so-called 'greenstick'- or 'trapdoor' orbital floor fracture, where entrapment of the inferior rectus muscle occurs due to the elastic bone in children. This is an indication of surgical intervention in less than 6 hours to prevent necrosis of this muscle which may cause lifelong vertical diplopia [19 - 21]. Enophtalmus and diplopia are the most common indications for later surgical repair in patients with orbital fractures (after 1-4 weeks, but preferably within 2 weeks) $[3,15,22]$.

Since 2015, our hospital has an integrated orbital trauma team at the outpatient clinic, where patients with an orbital fracture receive a combined appointment with the orthoptist, ophthalmologist and OMF surgeon. This collaborative approach was established because of the large proportion of facial trauma in our hospital and the need to standardise care for these patients. In the past, most of these patients would be seen either by an OMF surgeon and/or an ophthalmologist, but usually not on the same day. In the current protocol, the emergency specialist plans consultations on the same day with all three specialties. Because of this protocolized multidisciplinary approach, patients are analysed and followed up in a standardised manner.

There are many studies concerning the epidemiology and aetiology of maxillofacial trauma and orbital fractures, but most studies have been conducted in Asia. Moreover, there are no recent reports on the incidence and aetiology of orbital fractures in the Netherlands or in Western Europe. Therefore, this study aims to describe the incidence, aetiology, types and treatment of orbital wall fractures in our hospital over a period of three years.

\section{METHODS}

\subsection{Integrated Approach of the Orbital Trauma Team}

When a patient visits the ER at the Haaglanden Medical Centre with clinical symptoms of an orbital fracture and when confirmed by facial CT scan, the ER doctor will consult a member of the orbital trauma team (OMF surgeon or ophthalmologist). When no immediate intervention is necessary, the patient will visit the outpatient clinic of the orbital trauma team within a few days. A thorough examination by both the OMF surgeon, ophthalmologist and orthoptist will take place on one day, and a joint decision will be made whether the patient will be treated conservatively or surgically (usually within two weeks following the orbital trauma). The surgery is performed by the OMF surgeon in our hospital. All patients will be followed up by a member of the orbital trauma team at least twice. Indications for surgical intervention (within two weeks) are enophthalmos more than $2 \mathrm{~mm}$ and/or diplopia and the willingness of the patient to undergo surgical treatment.

\subsection{Study Population}

This study was conducted at Haaglanden Medical Centre, located at Westeinde at the Hague, the Netherlands. The medical records of patients with ICD-10 code 'orbital fracture' were reviewed at the Department of Ophthalmology between June 2015 and July 2018. Patients were excluded from participation in this study when there was an absence of orbital fracture on facial CT scan, when they did not visit the ophthalmology outpatient clinic for ophthalmological assessment, or when there was only one visit at the outpatient clinic following the trauma and no show on further follow-up visits (or telephone contact). Ophthalmological follow-up consisted of at least two outpatient visits. The outpatient visit at the OMF department was also conducted twice. Of these patients, information was also received from ER charts. The diagnosis 'orbital fracture' was based on a maxillofacial CT scan.

The collected data included gender, age, ophthalmologic examination after trauma, type of orbital fracture (including the site of injured eye), mechanism of trauma, modality of treatment (surgical or conservative), and intoxication during trauma. Mechanism of trauma was assigned to 5 categories: motor vehicle related (MVR) accident (pedestrian, bicycler, scooter/moped or electric bicycler), assault, sports, fall or 'other').

The ophthalmologic examination at the outpatient clinic consisted of visual acuity, examination of the anterior and posterior segment of the eye, analysis of the eye muscle motility, pupil reaction, examination of the sensibility of the infraorbital nerve, Hertel exophthalmometry and assessment of diplopia in all primary eye positions by the ophthalmologist 
and orthoptist.

We re-assessed our patient cohort according to the former treatment protocol (before the integrated approach). We based this analysis on the former practice styles of two of the older and one retired OMF surgeon from our hospital. We thereby could give a hypothetical estimate of the proportion of surgical intervention in the past (before 2010). The main difference between the former treatment protocol and the current integrated protocol is that previously most patients with prolapse of orbital fat and/or one or more extraocular muscles were operated regardless of diplopia or enophthalmos, and that ophthalmological assessment was not the standard procedure. In the present protocol, these patients are treated conservatively, when they do not have diplopia and/or enophthalmos more than $2 \mathrm{~mm}$ after two follow-up visits.

Furthermore, we reviewed the medical records of patients with maxillofacial injury at the Department of Oral and Maxillofacial Surgery between June 2015 and July 2018. Noted diagnosis included zygomatic fracture, maxillary sinus fracture, mandibular fracture, orbital fracture, nasal fracture, Le Fort I, II and III, facial contusion, dental injury and isolated soft tissue damage.

\subsection{Study Parameters}

The primary study parameters were types of orbital fractures and the treatment (conservative as well as surgical). Secondary outcomes were aetiology of orbital fractures, intoxication during the orbital trauma (alcohol or drug use at the time of the accident, according to the ER chart), and hypothetical intervention based on the former protocol.

\subsection{Ethics}

In the Netherlands, the Medical Research Involving Human Subjects Act (WMO) applies to all medical scientific research in which humans are subjected to procedures or follow rules of behaviour. All other research (with medical data or interviews) is not subjected to the WMO. The medical research ethics committee of our hospital was consulted, and a 'non-WMO declaration' was received, since this has been a retrospective observational study.

\subsection{Statistical Analysis}

All data were imported in Castor Electronic Data Capture, the data management system of our hospital. The data was analysed using SPSS version 25. Descriptive statistics was used to analyse patient characteristics. We analysed differences in baseline characteristics between the two groups using the independent $t$-test. Outcomes were considered statistically significant if the two-sided P-values were $<0.05$.

\subsection{Type of Study}

Retrospective analysis on the aetiology, types, clinical symptoms and treatment of orbital fractures of maxillofacial trauma was conducted in a period of three years at HMC Westeinde (the Netherlands).

\section{RESULTS}

Between June 2015 and July 2018, 314 patients were selected from the registration system of HMC Westeinde. Of them, 200 had an orbital wall fracture, according to their medical chart. After reviewing, 18 patients were excluded because there was no visible fracture on $\mathrm{CT}$ scan or because of loss to follow up.

In total, 182 patients were included with 287 fractured orbital walls. The mean age of the patients was 48.3 years, and $70.3 \%$ of the patients were male (Table 1). Female patients were significantly older than male patients ( 58.4 years versus 44.0 years, $\mathrm{p}<0.005)$. The outcomes of ophthalmological examination are presented in Table 2.

Table 1. Patient characteristics of 182 patients with an orbital fracture.

\begin{tabular}{|c|c|}
\hline Characteristic & $\mathbf{N}=\mathbf{1 8 2}$ \\
\hline Mean age at trauma (SD) & $48.3(21.9)$ \\
\hline Motor vehicle related accident & $48.9(18.2)$ \\
\hline Assault & $34.3(13.4)$ \\
\hline Sports accident & $23.7(22.3)$ \\
\hline Fall & $62.6(20.3)$ \\
\hline Gender & - \\
\hline Male (\%) & $128(70.3)$ \\
\hline Female (\%) & $54(29.7)$ \\
\hline
\end{tabular}

Table 2. Ophthalmologic examination.

\begin{tabular}{|c|c|}
\hline Examination & N $=\mathbf{1 8 2}$ \\
\hline Vision OD after trauma; mean (SD) & $0.9(0.24)$ \\
\hline Vision OS after trauma; mean (SD) & $0.9(0.27)$ \\
\hline Difference OD-OS Hertel in millimetre; mean (SD) & $0.88(0.98)$ \\
\hline Impaired eye motility (\%) & $39(21.4)$ \\
\hline Impaired sensitivity infraorbital nerve (\%) & $35(19.2)$ \\
\hline Impaired pupil reaction (\%) & $7(3.8)$ \\
\hline Impaired Ishihara test (\%) & $3(1.6)$ \\
\hline
\end{tabular}

In this period, 680 patients were seen at the OMF outpatient clinic with maxillofacial injury (Table 3). The most common injury was zygomatic fracture $(31.4 \%, \mathrm{n}=214)$, followed by orbital fracture $(29.2 \%, \mathrm{n}=198)$ and mandibular fracture $(17.5 \%, \mathrm{n}=119)$.

Table 3. Maxillofacial injury at the Department of Oral and Maxillofacial Surgery from June 2015 until July 2018.

\begin{tabular}{|c|c|}
\hline Diagnosis* & N = 680 (\%) \\
\hline Zygoma fracture & $214(31.4)$ \\
\hline Maxillary sinus fracture & $63(9.2)$ \\
\hline Mandibular fracture & $119(17.5)$ \\
\hline Orbital wall fracture & $198(29.2)$ \\
\hline Nasal fracture & $51(7.5)$ \\
\hline Le fort I & $21(3.0)$ \\
\hline Le fort II & $16(2.3)$ \\
\hline Le fort III & $13(1.9)$ \\
\hline Facial contusion & $47(6.9)$ \\
\hline Dental injury & $43(6.3)$ \\
\hline Isolated soft tissue injury & $14(2.0)$ \\
\hline$=$ multiple diagnosis possible &
\end{tabular}

*=multiple diagnosis possible. 
Table 4. Types of orbital fracture according to the injured walls, compared with performed surgical intervention and hypothetical surgical intervention before integration of the orbital trauma team.

\begin{tabular}{|c|c|c|c|c|c|}
\hline \multirow{2}{*}{$\begin{array}{c}- \\
\text { Type of fracture }\end{array}$} & \multirow{2}{*}{$\begin{array}{c}- \\
N(\%) \\
\end{array}$} & \multicolumn{2}{|c|}{ Surgical intervention* } & \multicolumn{2}{|c|}{ Hypothetical surgical intervention } \\
\hline & & Yes $(\%)$ & No (\%) & Yes (\%) & No (\%) \\
\hline Lateral wall (isolated) & $5(2.7)$ & $1(20)$ & $4(80)$ & $1(20)$ & $4(80)$ \\
\hline Lateral wall + zygoma & $13(7.1)$ & $5(38.5)$ & $8(61.5)$ & $10(76.9)$ & $3(23.1)$ \\
\hline Orbital roof (isolated) & $12(6.6)$ & $2(16.7)$ & $10(83.3)$ & $2(16.7)$ & $10(83.3)$ \\
\hline Orbital roof + medial wall & $9(4.9)$ & $0(0)$ & $9(100)$ & $1(11.1)$ & $8(88.9)$ \\
\hline Orbital roof + lateral wall & $2(1.1)$ & $0(0)$ & $2(100)$ & $1(50)$ & $1(50)$ \\
\hline Orbital roof, medial wall + lateral wall & $3(1.6)$ & $1(33.3)$ & $2(66.7)$ & $1(33.3)$ & $2(66.7)$ \\
\hline Orbital floor (isolated) & $56(30.8)$ & $16(28.6)$ & $40(71.4)$ & $35(62.5)$ & $21(37.5)$ \\
\hline Orbital floor + medial wall & $23(12.6)$ & $11(47.8)$ & $12(52.2)$ & $17(73.9)$ & $6(26.1)$ \\
\hline Orbital floor + zygoma & $15(8.2)$ & $7(46.7)$ & $8(53.3)$ & $10(66.7)$ & $5(33.3)$ \\
\hline Orbital floor, lateral wall + zygoma & $15(8.2)$ & $10(66.7)$ & $5(33.3)$ & $13(86.7)$ & $2(13.3)$ \\
\hline Orbital floor, medial wall + lateral wall & $2(1.1)$ & $1(50)$ & $1(50)$ & $2(100)$ & $0(0)$ \\
\hline Orbital floor + lateral wall & $5(2.7)$ & $1(20)$ & $4(80)$ & $2(40)$ & $3(60)$ \\
\hline Medial wall (+ nasal bone fracture) & $21(11.5)$ & $1(4.8)$ & $20(95.2)$ & $9(42.9)$ & $12(57.1)$ \\
\hline Zygomatic fracture (isolated) & $1(0.5)$ & $0(0)$ & $1(100)$ & $0(0)$ & $1(100)$ \\
\hline Total & $182(100)$ & $56(30.8)$ & $126(69.2)$ & $104(57.1)$ & $78(42.9)$ \\
\hline
\end{tabular}

* Indication for surgical intervention: enopthalmus $>2 \mathrm{~mm}$ and/or diplopia

${ }^{\delta}$ Hypothetical intervention based on former practice style of three OMF surgeons in our hospital: moderate to severe prolapse, with or without diplopia or enophtalmus $>2 \mathrm{~mm}$

\subsection{Types of Orbital Fractures and Treatment}

The most common type of orbital fracture was an isolated orbital floor fracture $(n=56,30.8 \%)$, followed by a combination of the orbital floor and medial wall fracture $(n=23,12.5 \%)$ (Table 4). The left eye was more often injured than the right eye $(50.5 \%, n=92$ versus $44 \%, n=80)$. In $5.5 \%(n=10)$ of the patients, both eyes were injured. The treatment of orbital fractures is presented in Table 5. Most patients with orbital fracture were treated conservatively $(69.2 \%, n=126)$. When surgical orbital wall reconstruction was performed, a PDS (polydioxanone) plate was used most frequently $(11.5 \%$, $\mathrm{n}=21)$. In 7 patients $(3.8 \%)$, an emergency canthotomy was performed to decompress the orbit. When we re-assessed our population based on the former protocol, we estimated that $57.1 \%(n=126)$ of the patients would be treated surgically (Table 4).

\subsection{Mechanism of Injury and Presence of Intoxication}

A fall was the main cause of orbital fractures $(38.5 \%$, $\mathrm{n}=70)$, followed by assault $(32.4 \%, \mathrm{n}=59)$ and motor vehicle related accidents $(24.2 \%, \mathrm{n}=44)$. In traffic, most patients were bicyclists $(65.9 \%, \mathrm{n}=29)$, followed by scooter/moped drivers $(22.7 \%, n=10)$ (Table 6). Fig. (1) illustrates the intoxications combined with the mechanisms of orbital trauma. In total, $32.4 \%(n=59)$ of the patients had a reported alcohol intoxication. Intoxication with drugs was reported in 4 patients.

Table 5. Treatment of 182 patients with an orbital fracture.

\begin{tabular}{|c|c|}
\hline Treatment & N=182 (\%) \\
\hline Conservative & $126(69.2)$ \\
\hline Zygomareposition without fixation & $12(6.6)$ \\
\hline PDS plate & $21(11.5)$ \\
\hline Zygomareposion with fixation & $18(9.9)$ \\
\hline Autologous bone transplantation & $2(1.1)$ \\
\hline Referral to academic centre & $3(1.6)$ \\
\hline
\end{tabular}

Table 6. Aetiology of orbital fractures.

\begin{tabular}{|c|c|}
\hline Cause & N=182 \\
\hline Motor vehicle related accident (\%) & $44(24.2)$ \\
\hline Pedestrian & 3 \\
\hline Bicycler & 29 \\
\hline Electric bicycler & 2 \\
\hline Scooter/moped & 10 \\
\hline
\end{tabular}


(Table 6) contd.....

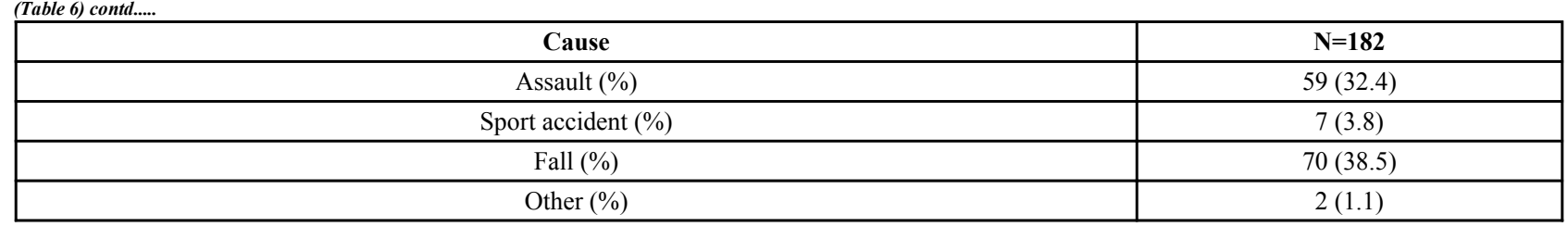

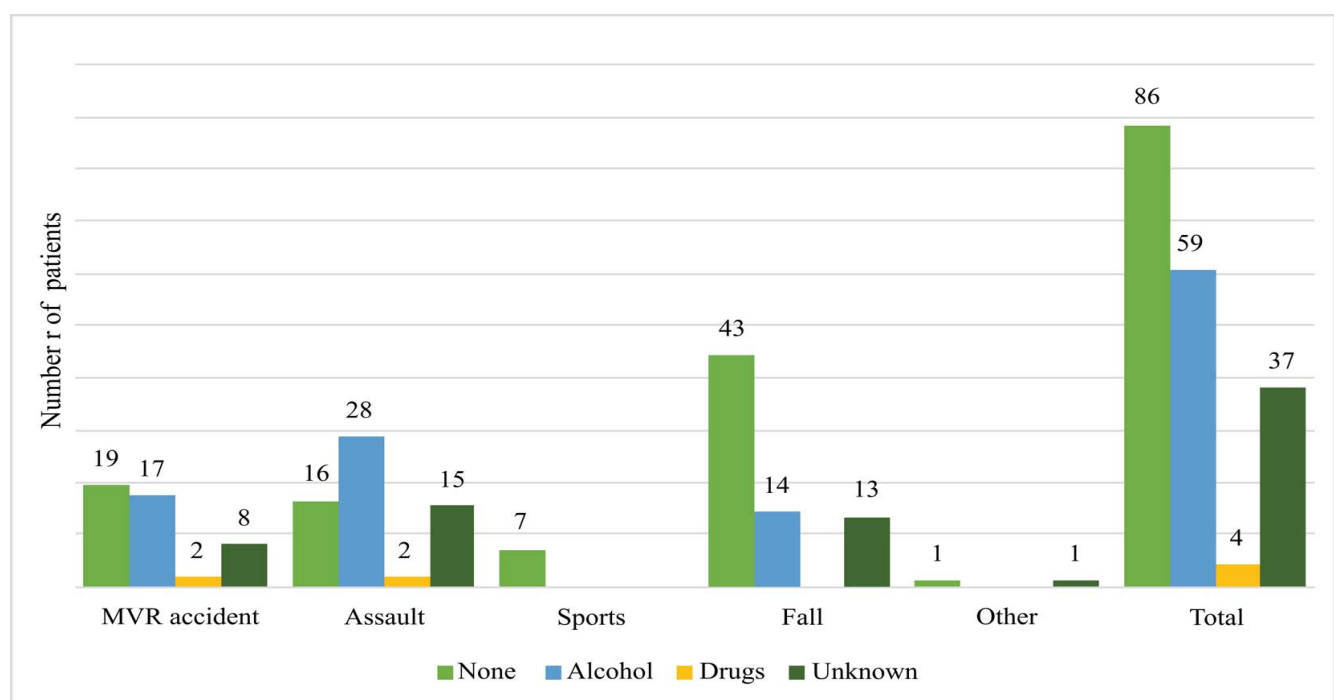

Fig (1). Intoxications of 182 patients with an orbital fracture.

\section{DISCUSSION}

This study provides more insight into the incidence, aetiology and treatment of orbital fractures in a large municipal hospital in the Netherlands. Between June 2015 and July 2018, 182 patients with an orbital fracture were seen by the orbital trauma team. Isolated orbital floor fractures were the most common type $(30.8 \%)$, and most patients were treated conservatively $(69.2 \%)$. In our study population, a fall was the main cause $(38.5 \%)$, and $32.4 \%$ of all patients were intoxicated with alcohol at the time of the injury. We found that since June $2015,91.1 \%(182 / 198)$ of the patients with an orbital fracture were seen by the collaborative team of OMF surgeon, ophthalmologist and orthoptist in our hospital.

In our study population, the mean age was 48.3 years, and most patients were male. In previous literature, most patients have been found to be younger [4 - 7, 9, 23]. However, this can be explained by the fact that in our population, a fall was the main cause of an orbital fracture, and the mean age of this group was 62.6 years. In the Netherlands, there are many elderly cyclists. The mean age of patients with an orbital fracture due to assault was 34.3 years, which is more comparable to previous literature. Also, we found that a fall was the main cause of orbital fracture $(38.5 \%)$, followed by assault $(32.4 \%)$, which is in contrast with most studies where assault is the main cause of orbital fractures [4, 7, 9, 23].

We found that $32.4 \%$ of the patients were intoxicated with alcohol. However, when examining separate groups of the mechanism of injury, we found that $38.6 \%$ of the patients due to MVR accidents had an alcohol intoxication. Moreover, $47.5 \%$ of the assault patients were intoxicated with alcohol, and in $25.4 \%$ of these patients, alcohol consumption was not reported, so this percentage could be even higher. Our results are in line with the literature concerning maxillofacial trauma and alcohol consumption, where higher rates of alcohol intoxication are found in patients due to assault [6, 14, 24, 25].

In our population, $3.8 \%$ showed signs of traumatic optic neuropathy. This is a remarkable detail, given the fact that the trauma had an impact significant enough to break the orbital walls; however, it still caused little damage to the eye and/or the optic nerve. Kreidl et al. suggest a protective mechanism of orbital fractures for ocular injury [26]. He et al. showed that traumatic optical neuropathy was associated with orbital blowout fractures in 3\% [27]. Previous literature shows that $22-29 \%$ of orbital trauma is associated with a finding of ocular injury [4, 26, 27]. Fortunately, most of the ocular injuries (mostly commotio retinae) are not severe and usually recover without any permanent damage [27].

In our study, the majority of the patients were treated conservatively (69.2\%). Previous studies show that $54.4 \%-63.2 \%$ of the patients with an orbital fracture were treated by surgical orbital wall reconstruction $[9,28,29]$. In the seventies, this percentage was $80 \%$ [30]. These percentages are considerably higher than that in our study. However, the hypothetical percentage of our study based on the former protocol would be $57.1 \%$, which is in line with these numbers. In our study, the integrated protocol mainly lowered the percentage of surgical intervention on the medial wall and orbital floor fractures.

A few limitations have to be considered. Firstly, the retrospective study design could possibly lead to selection bias. However, we found that since June 2015, 91.1\% (182/198) of the patients coded with the diagnosis 'orbital fracture', were 
seen by the collaborative team of OMF surgeon, ophthalmologist and orthoptist in our hospital. Secondly, all data was gathered from a single, large level 1 trauma centre. The case-mix might be different in other parts of the world. The presence of intoxication was only based on the subjective description in the charts and no blood alcohol and/or drug measurements were performed.

\section{CONCLUSION}

This study was the first large study on the epidemiology of orbital fractures in the Netherlands, which included both conservatively as well as surgically treated patients. We recommend a large multicentre study to confirm our results. Also, prevention of falls in the elderly may lead to a diminishment of orbital fractures in the elderly, and prevention of aggression and alcohol consumption in young men may lead to less orbital fractures caused by assault.

Another limitation is that we cannot provide a valid comparison between the surgical interventions based on the former and the current protocol. There are no historical control groups since there was a gradual evolution in the assessment of the patients with an orbital fracture; therefore, we based our hypothetical surgical intervention on interviews with three of the elderly OMF surgeons. An advantage of this is that the cases are similar in the re-assessment with a historical view.

We think that the integrated care path of the orbital trauma team has contributed to the low percentage of surgical intervention in this study. By bringing knowledge and experience of different disciplines together into one path of care, patients are analysed in a more efficient manner. We think that close cooperation between ophthalmologist, OMF surgeon and orthoptist can lead to a significant improvement in efficiency of the treatment of orbital fractures. Through the results of this study, we hope to motivate other hospitals to implement a multidisciplinary orbital trauma team for a more structured and thorough analysis of patients with orbital fractures and apply surgical intervention more efficiently.

\section{ETHICS APPROVAL AND CONSENT TO PARTICIPATE}

The medical research ethics committee of our hospital was consulted, and a 'non-WMO declaration' was received, since this has been a retrospective observational study.

\section{HUMAN AND ANIMAL RIGHTS}

Not applicable.

\section{CONSENT FOR PUBLICATION}

Not applicable.

\section{AVAILABILITY OF DATA AND MATERIALS}

The data supporting the findings of the article is available in the [Castor EDC] at [https://data.castoredc.com/].

\section{FUNDING}

None.

\section{CONFLICT OF INTEREST}

None.

\section{ACKNOWLEDGEMENTS}

Declared none.

\section{REFERENCES}

[1] Bord SP, Linden J. Trauma to the globe and orbit. Emerg Med Clin North Am 2008; 26(1): 97-123, vi-vii. [vi-vii.].

[http://dx.doi.org/10.1016/j.emc.2007.11.006] [PMID: 18249259]

[2] Boffano P, Roccia F, Zavattero E, et al. European Maxillofacial Trauma (EURMAT) project: a multicentre and prospective study. J Craniomaxillofac Surg 2015; 43(1): 62-70.

[http://dx.doi.org/10.1016/j.jcms.2014.10.011] [PMID: 25457465]

[3] Boyette JR, Pemberton JD, Bonilla-Velez J. Management of orbital fractures: challenges and solutions. Clin Ophthalmol 2015; 9: 2127-37. [http://dx.doi.org/10.2147/OPTH.S80463] [PMID: 26604678]

[4] Shere JL, Boole JR, Holtel MR, Amoroso PJ. An analysis of 3599 midfacial and 1141 orbital blowout fractures among 4426 United States Army Soldiers, 1980-2000. Otolaryngol Head Neck Surg 2004; 130(2): 164-70.

[http://dx.doi.org/10.1016/j.otohns.2003.09.018] [PMID: 14990911]

[5] Chi MJ, Ku M, Shin KH, Baek S. An analysis of 733 surgically treated blowout fractures. Ophthalmologica 2010; 224(3): 167-75. [http://dx.doi.org/10.1159/000238932] [PMID: 19776656]

[6] Chiang E, Saadat LV, Spitz JA, Bryar PJ, Chambers CB. Etiology of orbital fractures at a level I trauma center in a large metropolitan city. Taiwan J Ophthalmol 2016; 6(1): 26-31.

[http://dx.doi.org/10.1016/j.tjo.2015.12.002] [PMID: 29018706]

[7] Erdmann D, Follmar KE, Debruijn M, et al. A retrospective analysis of facial fracture etiologies. Ann Plast Surg 2008; 60(4): 398-403. [http://dx.doi.org/10.1097/SAP.0b013e318133a87b] [PMID: 18362568]

[8] Ellis E, El-Attar A, Moos KF. An analysis of 2,067 cases of zygomatico-orbital fracture. J Oral Maxillofac Surg 1985; 43(6): 417-28.

[http://dx.doi.org/10.1016/S0278-2391(85)80049-5] [PMID: 3858478]

[9] Hwang K, You SH, Sohn IA. Analysis of orbital bone fractures: a 12year study of 391 patients. J Craniofac Surg 2009; 20(4): 1218-23. [http://dx.doi.org/10.1097/SCS.0b013e3181acde01] [PMID: 19553835]

[10] Oppenheimer AJ, Monson LA, Buchman SR. Pediatric orbital fractures. Craniomaxillofac Trauma Reconstr 2013; 6(1): 9-20. [http://dx.doi.org/10.1055/s-0032-1332213] [PMID: 24436730]

[11] Losee JE, Afifi A, Jiang S, et al. Pediatric orbital fractures: classification, management, and early follow-up. Plast Reconstr Surg 2008; 122(3): 886-97.

[http://dx.doi.org/10.1097/PRS.0b013e3181811e48]

[PMID: 18766055]

[12] Laar M. Jaarbericht Nationale Drug Monitor 2017. Utrecht: Trimbos instituut 2018.

[13] Han SB, Yang HK, Woo SJ, Hyon JY, Hwang JM. Association of alcohol consumption with the risk of ocular trauma. J Korean Med Sci 2011; 26(5): 675-8.

[http://dx.doi.org/10.3346/jkms.2011.26.5.675] [PMID: 21532860]

[14] Salentijn EG, van den Bergh B, Forouzanfar T. A ten-year analysis of midfacial fractures. J Craniomaxillofac Surg 2013; 41(7): 630-6. [http://dx.doi.org/10.1016/j.jcms.2012.11.043] [PMID: 23419413]

[15] Alinasab B, Ryott $M$, Stjärne P. Still no reliable consensus in management of blow-out fracture. Injury 2014; 45(1): 197-202.

[http://dx.doi.org/10.1016/j.injury.2012.09.009] [PMID: 23141204]

[16] Cruz AA, Eichenberger GC. Epidemiology and management of orbital fractures. Curr Opin Ophthalmol 2004; 15(5): 416-21.

[http://dx.doi.org/10.1097/01.icu.0000136113.56288.87] [PMID: 15625903]

[17] Young SM, Kim YD, Kim SW, et al. Conservatively treated orbital blowout fractures: Spontaneous radiologic improvement. Ophthalmology 2018; 125(6): 938-44.

[http://dx.doi.org/10.1016/j.ophtha.2017.12.015] [PMID: 29398084]

[18] van der Wal KG, de Visscher JG. [Blow-out fractures of the orbit]. Ned Tijdschr Geneeskd 1989; 133(44): 2164-7.

[PMID: 2682287]

[19] Phan LT, Jordan Piluek W, McCulley TJ. Orbital trapdoor fractures. Saudi J Ophthalmol 2012; 26(3): 277-82. 
[http://dx.doi.org/10.1016/j.sjopt.2012.05.008] [PMID: 23961006]

[20] Kemps PG, Frank MH. Football causes orbital trapdoor fracture with restricted eye movement. Lancet 2020; 395(10221): 370 [http://dx.doi.org/10.1016/S0140-6736(19)33223-4]

[PMID: 32007171]

[21] Tan J, Simonsz HJ, Koudstaal MJ. [Blunt trauma to the eye in children: more than a black eye]. Ned Tijdschr Geneeskd 2012; 156(40): A5262.

[PMID: 23031241]

[22] Burnstine MA. Clinical recommendations for repair of isolated orbital floor fractures: an evidence-based analysis. Ophthalmology 2002; 109(7): 1207-0. discussion 10-1; quiz 12-3.

[23] Boffano P, Roccia F, Gallesio C, Karagozoglu KH, Forouzanfar T. Diplopia and orbital wall fractures. J Craniofac Surg 2014; 25(2): e183-5.

[http://dx.doi.org/10.1097/SCS.0000000000000437] [PMID: 24621766]

[24] Laverick S, Patel N, Jones DC. Maxillofacial trauma and the role of alcohol. Br J Oral Maxillofac Surg 2008; 46(7): 542-6.

[http://dx.doi.org/10.1016/j.bjoms.2008.01.021] [PMID: 18687507]

[25] Conway DI, McMahon AD, Graham L, et al. The scar on the face of Scotland: deprivation and alcohol-related facial injuries in Scotland. J
Trauma 2010; 68(3): 644-9

[http://dx.doi.org/10.1097/TA.0b013e3181a5ed18] [PMID: 19918199]

[26] Kreidl KO, Kim DY, Mansour SE. Prevalence of significant intraocular sequelae in blunt orbital trauma. Am J Emerg Med 2003; 21(7): 525-8.

[http://dx.doi.org/10.1016/j.ajem.2003.08.009] [PMID: 14655229]

[27] He D, Blomquist PH, Ellis E III. Association between ocular injuries and internal orbital fractures. J Oral Maxillofac Surg 2007; 65(4): 713-20.

[http://dx.doi.org/10.1016/j.joms.2006.09.006] [PMID: 17368368]

[28] Chen HH, Pan CH, Leow AM, Tsay PK, Chen CT. Evolving concepts in the management of orbital fractures with enophthalmos: A retrospective comparative analysis. Formosan Journal of Surgery 2016; 49(1): 1-8.

[http://dx.doi.org/10.1016/j.fjs.2015.06.002]

[29] Beigi B, Khandwala M, Gupta D. Management of pure orbital floor fractures: a proposed protocol to prevent unnecessary or early surgery. Orbit 2014; 33(5): 336-42.

[http://dx.doi.org/10.3109/01676830.2014.902475] [PMID: 24987818]

[30] Greenwald HS Jr, Keeney AH, Shannon GM. A review of 128 patients with orbital fractures. Am J Ophthalmol 1974; 78(4): 655-64. [http://dx.doi.org/10.1016/S0002-9394(14)76304-4] [PMID: 4411734]

(C) 2021 Kooger et al.

This is an open access article distributed under the terms of the Creative Commons Attribution 4.0 International Public License (CC-BY 4.0), a copy of which is available at: https://creativecommons.org/licenses/by/4.0/legalcode. This license permits unrestricted use, distribution, and reproduction in any medium, provided the original author and source are credited. 